\title{
Dell'uso del principio delle velocità virtuali con riguardo all'attrito.
}

\author{
(del D.r Giulio Petersen, a Copenhagen.)
}

\begin{abstract}
0 uando si cercano le posizioni d'equilibrio d'un sistema mediante le sei note equazioni, si devono effettuare in generale molte eliminazioni di pressioni e di tensioni incognite; dopo di che le equazioni che restano, combinate colle condizioni puramente geometriche cui è soggetto il sistema, danno le cercate posizioni d'equilibrio.

Si ottiene quasi sempre una grande semplificazione nei calcoli facendo uso del principio delle velocità virtuali, il quale fornisce tosto le equazioni che altrimenti non si potrebbero conseguire che coll'eliminazione delle forze incognite. Per meglio chiarire cio che segue, vogliamo dapprima esaminare più da vicino il modo in cui si raggiunge questo scopo.

La condizione generale dell' equilibrio d'un punto è che sía nulla la somma delle componenti delle forze attive secondo una direzione arbitraria; si pnò in tal modo, scegliendo più direzioni distinte, formare tante equazioni quante si vogliano; ma tre sole di queste sono indipendenti fra loro, e in pari tempo sufficienti a risolvere il problema. Queste equazioni hanno la forma

$$
\sum P_{k} \cos \alpha_{k}=0
$$

dove $P_{k}$ è una qualunque delle forze ed $\alpha_{k}$ l'angolo che la sua direzione forma con quella della retta secondo cui se ne prendono le componenti. Moltiplicando la precedente equazione per un piccolo segmento $m$ di questa retta si ottiene la condizione equivalente
\end{abstract}

$$
\Sigma m P_{k} \cos \alpha_{k}=0,
$$


ossia

$$
\mathbf{\Sigma} P_{k} p_{k}=0
$$

Considerando $m$ come uno spostamento del punto, $p_{k}$ diventa la projezione di questo spostamento sulla direzione della forza $P_{k}$, e, usando la solita denominazione per il prodotto $P_{k} p_{k}$, si conclude tosto che:

Affinchè un punto sia in equilibrio, dev'esser nulla per ogni suo spostamento la somma dei momenti virtuali delle forze attive.

Questo teorema non differisce dal primo che nella forma, griacchè dando al punto tre spostamenti arbitrari, non si fa che decomporre le forze secondo tre direzioni diverse, e le equazioni che si ottengono sono in ambedue $i$ casi le stesse. Alle forze ag'enti si devono poi sempre ascrivere tanto le forze esterne date, quanto quelle che vengono introdotte a titolo di pressioni, tensioni, ecc.

Perchè un dato sistema sia in equilibrio, è necessario e sufficiente che lo sia ciascuno dei suoi punti in particolare. Dando a cias cuno di questi punti tre spostamenti particolari si otterrebbe il necessario numero d'equazioni; ma queste conterrebbero le pressioni o tensioni incognite come se si facesse la decomposizione delle forze nel modo ordinario. Bisogna dunque dirigere gli spostamenti in modo che le forze incognite non entrino nelle equazioni; quando una di queste forze è per tal modo eliminata, si ha un'incognita di meno e si puo quindi far a meno di un'equazione, cioè d'uno spostamento.

Se manca soltanto una condizione a che le posizioni dei varii punti del sistema siano totalmente determinate (senza riguardo alle forze agenti, cioè in un senso puramente geometrico), ogni punto non pud percorrere che una linea; in questo caso le pressioni delle varie linee vengono neutralizzate dirigendo gli spostamenti secondo le tangenti di esse, poichè per ogni punto basta un solo spostamento. Se mancano due condizioni, ogni punto non puó percorrere che una superficie, e la reazione di questa superficie viene neutralizzata dirigendo due diversi spostamenti del punto corrispondente nel piano tangente della stessa. Si traduce analiticamente questo processo dando alle $x, y, z$ gli incrementi $d x, d y, d z$, dei quali i primi due sono arbitrari e il terzo dipende da questi nel modo che risulta differenziando l'equazione della superficie. Per un artificio analitico assai semplice, invece di stabilire due equazioni, relative a due spostamenti, se ne scrive una sola, 
la quale, per l'indipendenza di $d x$ e $d y$, ne comprende realmente due distinte.

In tal modo si sono neutralizzate le reazior i incognite, rendendo in pari tempo determinate le direzioni degli spostamenti; ma i rapporti di grandezza di questi spostamenti rimangono tuttavia arbitrari. Si può quindi disporre di essi in modo da neutralizzare anche le tensioni incognite, per guisa che le equazioni contengano soltanto le forze esterne date.

Quando lungo una linea retta agiscono due forze eguali e contrarie applicate ai suoi estremi, è facile dimostrare che la somma dei loro momenti virtuali è nulla per ogni variazione infinitesima della retta che ne lasci invariata la lunghezza. Ora, risultando appunto ogni tensione dalla presenza di due forze eguali e contrarie, e chiaro che i relativi momenti entreranno nelle equazioni per modo da poter essere eliminati per via di addizione, se le grandezze degli spostamenti (già determinati quanto alla direzione) si faranno dipendere le une dalle altre al modo stesso che lo sarebbero per un moto infinitesimo puramente geometrico, soddisfacente alle condizioni prescritte; vale a dire se si stabiliranno fra le variazioni delle coordinate quelle stesse relazioni che risultano dalla differenziazione delle date equazioni.

Cosi si riesce a far dipendere la determinazione delle posizioni d'equilibrio di un dato sistema dalla considerazione delle sole forze esterne. Sfortunatamente questo metodo non puo essere applicato immediatamente quando si deve tener conto dell'attrito, giacchè le forze procedenti da questa causa non escono dal calcolo, e le pressioni incognite da cui dipendono ricompajono con esse nelle equazioni. Con ciò il principio perde molta della sua utilitá, non potendo venir applicato nella pratica, dove il più delle volte si deve aver riguardo agli attriti.

Noi ci proponiamo di mostrare tuttavia che il metodo può essere esteso anche a questo caso. Ci occuperemo dapprima di una figura piana, e passeremo poscia al caso generale.

Sia $P$ la pressione in un punto dove ha luogo attrito. La forza generata dall'attrito è $\mu P, \mu$ essendo il coefficiente d'attrito, ed è diretta secondo la tangente. Queste due forze si possono comporre in una sola di grandezza $P \sqrt{1+\mu^{2}}$, la cui direzione fa con quella della normale un angolo $v$ dato da

$$
\operatorname{tg} v=\mu \text {. }
$$

Rammentando ora che la pressione $P$ è stata precedentemente neutraliz- 
zata con uno spostamento diretto secondo la tangente, è chiaro che per neutralizzare la nuova forza bisognerà dirigere le spostamento perpendicolarmente alla sua direzione, cioè in modo da formare un angolo $v$ colla tangente alla linea percorsa dal punto. Dunque il principio delle velocita virtuali puo essere applicato anche quando si debba tener conto dell'attrito, purchè gli spostamenti dei punti dov'esso si sviluppa formino colla tangente un angolo la cui tangente trigonometrica sia eguale al coefficiente d'attrito.

Gli altri spostamenti dipendono da questi nello stesso modo di prima, perchè le rette secondo le quali si operano le tensioni devono restare di lunghezza costante, affinchè spariscano i momenti virtuali delle tensioni stesse.

Quando la sola forza esterna è la gravità, indicando i pesi con $P$, e le altezze dei singoli centri di gravità con $y$, si ha

$$
P d y+P_{1} d y_{1} \ldots+P_{n} d y_{n}=0,
$$

ossia, chiamando $\eta$ l'altezza del centro di gravità generale,

$$
d \eta=0 \text {, }
$$

donde si conclude che nelle posizioni d'equilibrio l'altezza del centro di gravità è un massimo o un minimo, semprechè le posizioni contigue risultino determinate da spostamenti nelle direzioni suindicate.

Chiameremo linea d'attrito la direzione secondo cui si deve concepire eseguito lo spostamento affinchè sia neutralizzata la risultante precedentemente considerata. Come abbiamo veduto, essa fa un angolo $v$ colla tangente.

Per trovare la formola da applicarsi per il calcolo, indichiamo con $x, y$ le coordinate di un punto dove abbia luogo attrito, e con $L=0$, l'equazione della linea su cui esso deve mantenersi. Siano $\delta x, \delta y$ gli incrementi delle coordinate relativi ad uno spostamento lungo la linea d'attrito. Siccome l'angolo di queste due direzioni è eguale a $v$ (essendo $\operatorname{tg} v=\mu$, dove $\mu$ può essere tanto positivo quanto negativo, poichè in generale non si può stabilire a priori la direzione della resistenza dovuta all'attrito), si ha

$$
\frac{\delta y}{\delta x}=\frac{\frac{d y}{d x}+\mu}{1-\mu \frac{d y}{d x}}=\frac{d y+\mu d x}{d x-\mu d y} .
$$


Ma dalla $L=0$ si trae

$$
\frac{\partial L}{\partial x} \delta x+\frac{\partial I}{\partial y} \delta y=0
$$

quindi, sostituendo per $\delta y: \delta x$ il valore dato dalla (1), si ha

$$
\left(\frac{\partial L}{\partial x}+\mu \frac{\partial L}{\partial y}\right) d x+\left(\frac{\partial L}{\partial y}-\mu \frac{\partial L}{\partial x}\right) d y=0
$$

Spostando $V_{1}$ lungo la linea d'attrito per un tratto avente la projezione $d x_{1}$, si ha

$$
d y_{1}=\mu_{1} d x_{1} .
$$

Il centro di $V$ viene con cid innalzato per un tratto $d y_{1}$, ma retrocede lungo la linea d'attrito per un tratto la cui projezione è $d x_{1}$, e poichè questa linea fa angoli eguali colla tangente e con un'orizzontale, si ha

$$
d y=d y_{1}-\mu d x_{1}
$$

di qui emerge per la condizione cercata

$$
2 V_{1}=\left(\frac{\mu}{\mu_{1}}-1\right) V
$$

Cerchiamo ora la formola generale da adoperarsi nel caso d'una superficie che presenti una resistenza dovuta all'attrito.

In luogo della linea d'attrito bisogna considerare in questo caso un cono di rotazione, il cui asse è la normale nel punto considerato, e il cui semiangolo al vertice è il complemento di $v$. Indicando con $L=0$ l'equazione della superficie e con $\alpha_{1}, \alpha_{2}, \alpha_{3}$ gli angoli della normale cogli assi, si ha

$$
\cos \alpha_{1}=\frac{\partial L}{\partial x} V, \quad \cos \alpha_{2}=\frac{\partial L}{\partial y} V, \quad \cos \alpha_{3}=\frac{\partial L}{\partial z} V
$$

dove

$$
V=\left\{\left(\frac{\partial L}{\partial x}\right)^{2}+\left(\frac{\partial L}{\partial y}\right)^{2}+\left(\frac{\partial L}{\partial z}\right)^{2}\right\}^{-\frac{1}{2}} .
$$

Indicando inoltre con $d x, d y, d z$ gli incrementi delle coordinate secondo una generatrice del cono e con $\sigma_{1}, b_{2}, b_{3}$ gli angoli di questa generatrice cogli assi, si ha

$\cos \varepsilon_{1}=\frac{d x}{\sqrt{d x^{2}+d y^{2}+d z^{2}}}, \quad \cos \varepsilon_{2}=\frac{d y}{\sqrt{d x^{2}+d y^{2}+d z^{2}}}, \quad \cos \varepsilon_{3}=\frac{d z}{\sqrt{d x^{2}+d y^{2}+d z^{2}}}$. 
Ma essendo

$$
\cos \left(90^{\circ}-v\right)=\cos \alpha_{1} \cos 6_{1}+\cos \alpha_{2} \cos 6_{2}+\cos \alpha_{3} \cos 6_{3}
$$

si ha

$$
\frac{\mu}{\sqrt{1+\mu^{2}}}=\frac{\frac{\partial L}{\partial x} d x+\frac{\partial L}{\partial y} d y+\frac{\partial L}{\partial z} d z}{\sqrt{\left\{\left(\frac{\partial L}{\partial x}\right)^{2}+\left(\frac{\partial L}{\partial y}\right)^{2}+\left(\frac{\partial L}{\partial z}\right)^{2}\right\}\left\{d x^{2}+d y^{2}+d z^{2}\right\}}}
$$

e questa è l'equazione che deve prendere il posto della (2) quando lungo la linea $L$ abbia luogo attrito. E d'uopo notare che siccome il punto non vien fatto muovere sulla linea data, bisogna prescindere da questa supposizione anche nel differenziare le equazioni di condizione, allo scopo di esprimere le dipendenze fra le variazioni delle coordinate dei diversi punti. Per es. se un punto è obbligato a restare sull'asse delle $x$, bisogna ció non ostante rappresentarlo con $(x, y)$ e fare $y=0$ soltanto nelle eliminazioni finali.

\section{Esempio $10^{\circ}$}

Una retta di lunghezza $l$ e di peso $V$ si appoggia colle sue estremitá contro gli assi coordinati; si deve determinare la posizione d'equilibrio, nella supposizione che i coefficienti d'attrito alle due estremità siano $\mu_{1}$ e $\mu_{2}$.

Sia $(x, y)$ il centro di gravità, $\left(x_{1}, y_{1}\right)$ l'estremità inferiore col coefficiente d'attrito $\mu_{1},\left(x_{2}, y_{2}\right)$ l'estremitá superiore col coefficiente d'attrito $\mu_{2}$. Si ha

Dalla

$$
\begin{array}{lcl}
y_{1}=0 & \text { donde } & \mu_{1} d x_{1}+d y_{1}=0 \\
x_{2}=0 & \gg & d x_{2}-\mu_{2} d y_{2}=0 \\
V d y=0 & \text { ossia } & d y_{1}+d y_{2}=0 .
\end{array}
$$

$$
\left(x_{1}-x_{2}\right)^{2}+\left(y_{1}-y_{2}\right)^{2}=l^{2}
$$

si deduce

$$
\left(x_{1}-x_{2}\right)\left(d x_{1}-d x_{2}\right)+\left(y_{1}-y_{2}\right)\left(d y_{1}-d y_{2}\right)=0
$$

epperò

$$
\operatorname{tg} \phi=\frac{y_{1}-y_{2}}{x_{1}-x_{2}}=-\frac{d x_{1}-d x_{2}}{d y_{1}-d y_{2}}=\frac{1-\mu_{1} \mu_{2}}{2 \mu_{1}}
$$

dove $\phi$ è l'inclinazione della retta $l$ nella cercata posizione d'equilibrio. 


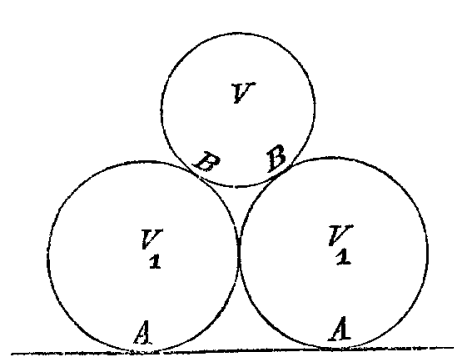

\section{Esempio $2 .^{\circ}$}

Abbiansi tre cilindri disposti nel modo indicato dalla figura; trovare la condizione perchè essi incomincino simultaneamente a strisciare in tutti i punti di contatto. Due abbiano il peso $V_{1}$, il terzo $V$; il coefficiente d'attrito sia $\mu_{1}$ in $A$ e $\mu$ in $B$.

Se si fa girare un poco uno dei cilindri intorno ad $A$, si scorge che la pressione totale in $B$ dev'essere diretta verso $A$, poichè in questo solo caso il suo momento virtuale puó essere eliso; la linea d'attrito è quindi perpendicolare a $B A$ epperò divide per metà l'angolo della tangente in $B$ con un'orizzontale. Indicando con $y_{1}$ ed $y$ le altezze dei centri si ha

$$
2 V_{1} d y_{1}+V d y=0 \text {. }
$$

ossia

$$
\begin{gathered}
\frac{\partial L}{\partial x} d x+\frac{\partial L}{\partial y} d y+\frac{\partial L}{\partial z} d z= \\
=\mu \sqrt{\left(\frac{\partial L}{\partial y} d z-\frac{\partial L}{\partial z} d y\right)^{z}+\left(\frac{\partial L}{\partial z} d x-\frac{\partial L}{\partial x} d z\right)^{2}+\left(\frac{\partial L}{\partial x} d y-\frac{\partial L}{\partial y} d x\right)^{2}},
\end{gathered}
$$

epperò questa è l'equazione cercata, da adoperarsi per le superficie nel caso dell'attrito. Per $\mu=0$ essa si riduce all'ordinaria condizione

$$
\frac{\partial L}{\partial x} d x+\frac{\partial L}{\partial y} d y+\frac{\partial L}{\partial z} d z=0
$$

per una linea esistente nel piano $x y$ si ha $z=0, d z=0, \frac{\partial L}{\partial z}=0$, e si ritrova la formola già precedentemente stabilita per questo caso particolare.

Analoghe considerazioni si possono spesso applicare vantaggiosamente anche in dinamica. Per conseguire la relativa formola cercheremo un'espressione della forza acceleratrice secondo la linea d'attrito, limitandoci, per evitare una soverchia complicazione, a considerare il caso in cui tanto la trajettoria, quanto le direzioni delle forze attive, esistano nel piano $x y$.

Le forze acceleratrici sono $\frac{d v}{d t}$ nel senso della tangente, $\frac{v^{2}}{p}$ nel senso della normale, oltre la pressione della linea e la resistenza dovuta all'attrito. Le due ultime danno una componente nulla nel senso della linea d'attrito, mentre 
le prime due danno, nello stesso senso, la componente

$$
\frac{1}{\sqrt{\tau+\mu^{2}}}\left(\frac{d v}{d t}+\mu \frac{v^{3}}{\rho}\right)
$$

dove il radicale deve prendersi positivamente se s'intende scelta la direzione positiva della linea d'attrito in modo da formare un angolo acuto colla direzione del moto.

a) Quando non agiscono forze esterne si ha

$$
\frac{d v}{d t}+\mu \frac{v^{2}}{\rho}=0
$$

ovvero, sostituendo il valore $\rho=\frac{d s}{d \theta}=v \frac{d t}{d \theta}$,

$$
\frac{d v}{v}+\mu d \theta=0
$$

donde

$$
v=v_{0} e^{-\mu \theta}
$$

dove $\theta$ è l'angolo della tangente coll'asse delle $x$, e $v_{0}$ la velocitá corrispondente al valore $\theta=0$. Supponendo $\rho$ e quindi $d \theta$ positivo, l'angolo $\theta$ è crescente, epperd la formola sussiste finchè non si giunga ad un punto di flesso; quando il punto passa sulla parte convessa della linea, la pressione diventa negativa, e il problema perde il suo significato.

b) Quando la forza esterna è la gravitả, la sua componente secondo la linea d'attrito è

$$
-\frac{g}{\sqrt{1+\mu^{2}}} \frac{d y+\mu a \vec{a} x}{d s}
$$

eppero l'equazione del moto diventa

$$
\frac{d v}{d t}+\mu v \frac{d \theta}{d t}=-g \frac{d y+\mu d x}{d s}
$$

ossia

$$
d \cdot v^{2}+2 \mu v^{2} d \theta=-2 g(\operatorname{sen} \theta+\mu \cos \theta) d s,
$$

donde

$$
v^{2}=-2 g e^{-2 \mu \theta}\left[\int e^{2 \mu \theta}(\operatorname{sen} \theta+\mu \cos \theta) d s+C\right] .
$$


94 Petersen: Dell'uso del principio delle velocità ecc.

Il problema è cosi ridotto ad una quadratura, qualora si esprima $\frac{d s}{d \theta}$ ossia $\rho$ in funzione di $\theta$. si ha

c) Quando le componenti delle forze secondo gli assi sono $X$ ed $Y$,

$$
d \cdot v^{2}+2 \mu v^{2} d \theta=\{2 X(\cos 0-\mu \operatorname{sen} \theta)+2 Y(\operatorname{sen} \theta+\mu \cos \theta)\} d s,
$$

equazione che può essere integrata come la precedente, quando $X$ ed $Y$ dipendano soltanto da $x$ ed $y$.

Copenhagen, novembre 1869. 